B. Ichim and T. Römer

Nagoya Math. J.

Vol. 194 (2009), 69-90

\title{
ON CANONICAL MODULES OF TORIC FACE RINGS
}

\author{
BOGDAN ICHIM AND TIM RÖMER
}

\begin{abstract}
Generalizing the concepts of Stanley-Reisner and affine monoid algebras, one can associate to a rational pointed fan $\Sigma$ in $\mathbb{R}^{d}$ the $\mathbb{Z}^{d}$-graded toric face ring $K[\Sigma]$. Assuming that $K[\Sigma]$ is Cohen-Macaulay, the main result of this paper is to characterize the situation when its canonical module is isomorphic to a $\mathbb{Z}^{d}$-graded ideal of $K[\Sigma]$. From this result several algebraic and combinatorial consequences are deduced. As an application, we give a relation between the cleanness of $K[\Sigma]$ and the shellability of $\Sigma$.
\end{abstract}

\section{$\S 1$. Introduction}

Let $\Sigma$ be a rational pointed fan in $\mathbb{R}^{d}$, i.e. $\Sigma$ is a finite collection of rational pointed cones in $\mathbb{R}^{d}$ such that for $C^{\prime} \subseteq C$ with $C \in \Sigma$ we have that $C^{\prime}$ is a face of $C$ if and only if $C^{\prime} \in \Sigma$, and if $C, C^{\prime} \in \Sigma$, then $C \cap C^{\prime}$ is a common face of $C$ and $C^{\prime}$. Stanley constructed in [20] the toric face ring $K[\Sigma]$ over a field $K$ associated to $\Sigma$ as follows.

As a $K$-vector space $K[\Sigma]=\bigoplus_{a \in \cup_{C \in \Sigma} C \cap \mathbb{Z}^{d}} K x^{a}$. The multiplication in $K[\Sigma]$ is defined by:

$$
x^{a} \cdot x^{b}= \begin{cases}x^{a+b} & \text { if } a \text { and } b \text { are elements of a common face } C \in \Sigma, \\ 0 & \text { otherwise. }\end{cases}
$$

We see that $K[\Sigma]$ is naturally a $\mathbb{Z}^{d}$-graded $K$-algebra. This class of rings generalizes the concepts of Stanley-Reisner rings associated to simplicial complexes and affine monoid algebras associated to affine monoids in some $\mathbb{Z}^{d}$. (See Bruns-Herzog [9] or Stanley [22] for a detailed discussion on these two special cases.)

Motivated by results from the two special cases, the authors started to study toric face rings systematically to obtain their algebraic properties in [15]. (See also [4], [5], [6], [8], [12], [17] and [23] for related results.)

Received June 21, 2007.

Revised June 26, 2008.

Accepted July 31, 2008.

2000 Mathematics Subject Classification: Primary 13C14, 13D45; Secondary 05E99. 
Two of the main results in [15] classify completely in which cases $K[\Sigma]$ is Cohen-Macaulay, respectively Gorenstein, in combinatorial terms of the rational pointed fan $\Sigma$. It is well-known that the Gorenstein property is equivalent to the fact that $K[\Sigma]$ is Cohen-Macaulay and there exists a $\sigma \in \mathbb{Z}^{d}$ such that the principal ideal $K[\Sigma](-\sigma)$ is isomorphic to $\omega_{K[\Sigma]}$ as a $\mathbb{Z}^{d}$ graded $K[\Sigma]$-module, where $\omega_{K[\Sigma]}$ denotes the $\mathbb{Z}^{d}$-graded canonical module of $K[\Sigma]$. Assuming only that $K[\Sigma]$ is Cohen-Macaulay, it is a natural question in which cases $\omega_{K[\Sigma]}$ is isomorphic to a $\mathbb{Z}^{d}$-graded ideal of $K[\Sigma]$. After introducing definitions and results related to toric face rings in Section 2, we give a complete characterization of this situation in Section 3 .

Intersecting each cone of $\Sigma$ with the unit sphere of $\mathbb{R}^{d}$ one obtains a regular cell complex $\Gamma_{\Sigma}$. In Section 4 we study algebraic properties of $K[\Sigma]$ in terms of topological properties of $\Gamma_{\Sigma}$. As in the case of simplicial complexes, the property of being Cohen-Macaulay is a topological property.

In particular, in Section 4, we get as an application of our main result of Section 3 the following theorem which generalizes a nice result of Hochster for Stanley-Reisner rings.

THEOREM 1.1. Let $\Sigma$ be a rational pointed fan in $\mathbb{R}^{d}$ such that $K[\Sigma]$ is a Cohen-Macaulay ring. Assume that $X=\left|\Gamma_{\Sigma}\right|$ is a manifold with a nonempty boundary $\partial X$. Further let $\Sigma^{\prime}$ be the subfan of $\Sigma$ such that $\partial X=\left|\Gamma_{\Sigma^{\prime}}\right|$. Then the following conditions are equivalent:

(i) $\omega_{K[\Sigma]}$ is isomorphic as a $\mathbb{Z}^{d}$-graded module to the kernel of the natural surjective homomorphism $K[\Sigma] \rightarrow K\left[\Sigma^{\prime}\right]$;

(ii) $K\left[\Sigma^{\prime}\right]$ is Gorenstein and $\omega_{K\left[\Sigma^{\prime}\right]} \cong K\left[\Sigma^{\prime}\right]$ as $\mathbb{Z}^{d}$-graded modules.

For the definition of an Euler fan we refer to Section 2. Condition (ii) of the theorem is equivalent to the fact that $K\left[\Sigma^{\prime}\right]$ is Cohen-Macaulay and $\Sigma^{\prime}$ is an Euler fan.

Recall that a linear ordering $C_{1}, \ldots, C_{s}$ of the facets of $\Sigma$ is called a shelling of $\Sigma$ if either $\operatorname{dim} \Sigma=0$ (and thus there is just one facet and that is a point), or $\operatorname{dim} \Sigma \geq 1$ and the following two conditions are satisfied:

(i) $\operatorname{Fan}\left(\partial C_{1}\right)$ has a shelling.

(ii) For $1<j \leq s$ there exists a shelling $D_{1}^{j}, \ldots, D_{t_{j}}^{j}$ of the (pure) fan $\operatorname{Fan}\left(\partial C_{j}\right)$ such that $\bigcup_{i=1}^{j-1} \operatorname{Fan}\left(C_{i}\right) \cap \operatorname{Fan}\left(C_{j}\right)$ is not empty and equals $\bigcup_{l=1}^{r_{j}} \operatorname{Fan}\left(D_{l}^{j}\right)$ for some integer $r_{j}$ with $1 \leq r_{j} \leq t_{j}$. 
$\Sigma$ is called shellable if it has a shelling. Remark that by the Bruggesser-Mani Theorem condition (i) of the above definition is in fact redundant (see [24, Chapter 8]). In the last Section of this paper we study algebraic properties of the toric face rings $K\left[\bigcup_{i=1}^{j-1} \operatorname{Fan}\left(C_{i}\right) \cap \operatorname{Fan}\left(C_{j}\right)\right]$ under the assumption that $\Sigma$ is shellable. We can prove that they are all Cohen-Macaulay and using the result of Section 4 mentioned above, we show that their canonical modules are isomorphic to $\mathbb{Z}^{d}$-graded ideals of the base rings. Moreover, this result holds also under the weaker assumption "semishellable" as defined in Section 5.

For a Stanley-Reisner ring $K[\Delta]$ corresponding to a simplicial complex $\Delta$, Dress [11] proved that the shellability of $\Delta$ is equivalent to the fact that $K[\Delta]$ is a clean ring (see Section 5 for the definition). This result does not hold for toric face rings. In fact, the main result of last section is the following theorem which generalizes the result of Dress.

THEOREM 1.2. Let $\Sigma$ be a rational pointed fan in $\mathbb{R}^{d}$. Then $K[\Sigma]$ is clean if and only if the following conditions are satisfied:

(i) $\Sigma$ is (non-pure) shellable with shelling $C_{1}, \ldots, C_{s}$.

(ii) For $1<j \leq s$ there exists $\gamma_{j} \in C_{j} \cap \mathbb{Z}^{d}$ such that

$$
\operatorname{star}_{\operatorname{Fan}\left(C_{j}\right)}\left(\gamma_{j}\right)=\operatorname{Fan}\left(C_{j}\right) \backslash\left(\bigcup_{i=1}^{j-1} \operatorname{Fan}\left(C_{i}\right)\right) .
$$

$$
\bigcup_{D \in \operatorname{star}_{\operatorname{Fan}\left(C_{j}\right)}\left(\gamma_{j}\right)} \operatorname{relint}(D) \cap \mathbb{Z}^{d}=\gamma_{j}+C_{j} \cap \mathbb{Z}^{d} .
$$

We are grateful to Prof. W. Bruns for inspiring discussions on the subject of the paper.

\section{$\S 2$. Toric face rings}

In this section we fix some notation and recall important results needed in the rest of the paper. Let $\Sigma$ be a rational pointed fan in $\mathbb{R}^{d}$. Let $K$ be a field. We consider the toric face ring $K[\Sigma]$ of $\Sigma$ over $K$ as defined in Section 1. For $C \in \Sigma$ we define the $\mathbb{Z}^{d}$-graded ideal $\mathfrak{p}_{C}=\left(x^{a} \in K[\Sigma]: a \notin C\right)$. It is easily verified that $K[\Sigma] / \mathfrak{p}_{C} \cong K[\operatorname{Fan}(C)]$ where $\operatorname{Fan}(C)$ is the fan consisting of all cones $D \in \Sigma$ such that $D \subseteq C$. In particular, $\mathfrak{p}_{C}$ is a prime ideal. More generally, for a subfan $\Sigma^{\prime} \subseteq \Sigma$ we set $\mathfrak{q}_{\Sigma^{\prime}}=\mathfrak{q}_{\Sigma^{\prime}}^{\Sigma}=\left(x^{a} \in K[\Sigma]: a \notin\left|\Sigma^{\prime}\right|\right)$. 


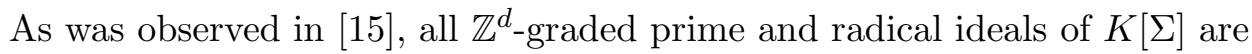
described as follows.

Lemma 2.1. Let $\Sigma$ be a rational pointed fan in $\mathbb{R}^{d}$.

(i) The assignment $C \mapsto \mathfrak{p}_{C}$ is a bijection between the set of non-empty cones in $\Sigma$ and the set of $\mathbb{Z}^{d}$-graded prime ideals of $K[\Sigma]$.

(ii) The assignment $\Sigma^{\prime} \mapsto \mathfrak{q}_{\Sigma^{\prime}}$ is a bijection between the set of non-empty subfans of $\Sigma$ and the set of $\mathbb{Z}^{d}$-graded radical ideals of $K[\Sigma]$.

In particular, $\mathfrak{m}=\mathfrak{p}_{0}$ is the unique $\mathbb{Z}^{d}$-graded maximal ideal of $K[\Sigma]$, which is also maximal in the usual sense.

For $C \in \Sigma$ let $\operatorname{star}_{\Sigma}(C)=\{D \in \Sigma: C \subseteq D\}$ be the star of $C$. In particular, $\operatorname{star}_{\Sigma}(0)=\Sigma$. Moreover, we set $\Sigma(C)=\Sigma \backslash \operatorname{star}_{\Sigma}(C)$. Observe that this a subfan of $\Sigma$.

We define a complex

$$
\mathscr{C}_{\operatorname{star}_{\Sigma}(C)}: 0 \longrightarrow \mathscr{C}^{\operatorname{dim} C-1} \stackrel{\partial}{\longrightarrow} \mathscr{C}^{\operatorname{dim} C} \longrightarrow \cdots \stackrel{\partial}{\longrightarrow} \mathscr{C}^{\operatorname{dim} \Sigma-1} \longrightarrow 0
$$

where

$$
\mathscr{C}^{i}=\bigoplus_{D \in \operatorname{star}_{\Sigma}(C), \operatorname{dim} D=i+1} K D
$$

for $i=\operatorname{dim} C-1, \ldots, \operatorname{dim} \Sigma-1$, and the differential is induced by an incidence function $\varepsilon$ on $\Sigma$. (See [15, Section 4] for details. There $\mathscr{C}_{\operatorname{star}_{\Sigma}(C)}$ was denoted by $\mathscr{C}_{\bullet}^{\bullet}\left(\Gamma_{\operatorname{star}_{\Sigma}(C)}\right)$.) Note that the (co-)homology $\widetilde{H}^{i}\left(\mathscr{C}_{\operatorname{star}_{\Sigma}(C)}\right)$ of the complex $\mathscr{C}_{\text {star }_{\Sigma}(C)}$ computes up to a shift the reduced cohomology groups $\widetilde{H}^{i}\left(\Delta\left(\operatorname{star}_{\Sigma}(C)\right)\right)$ with coefficients in $K$ of the order complex $\Delta\left(\operatorname{star}_{\Sigma}(C)\right)$ of the poset $\operatorname{star}_{\Sigma}(C)-\{C\}$. Indeed, in [15, Lemma 4.6] it was shown that $\widetilde{H}^{i}\left(\mathscr{C}_{\operatorname{star}_{\Sigma}(C)}\right)=\widetilde{H}^{i-\operatorname{dim} C}\left(\Delta\left(\operatorname{star}_{\Sigma}(C)\right)\right)$ for all integers $i$.

Observe that for $a \in \operatorname{relint}(C) \cap \mathbb{Z}^{d}$ we have $\operatorname{star}_{\Sigma}(C)=\{D \in \Sigma: a \in$ $D\}$. Hence it makes sense to define slightly more generally for $a \in \mathbb{Z}^{d}$ the star of $a$ as $\operatorname{star}_{\Sigma}(a)=\{D \in \Sigma: a \in D\}$. Analogously we define $\Sigma(a)$ and the complex $\mathscr{C}_{\operatorname{star}_{\Sigma}(a)}$. By the local cohomology groups of $K[\Sigma]$ we always mean the local cohomology with respect to the $\mathbb{Z}^{d}$-graded maximal ideal $\mathfrak{m}$ of $K[\Sigma]$. A Hochster type formula for the local cohomology of toric face rings is given by: 
Theorem 2.2. ([15, Corollary 4.7]) Let $\Sigma$ be a rational pointed fan in $\mathbb{R}^{d}$. Then

$$
H_{\mathfrak{m}}^{i}(K[\Sigma]) \cong \bigoplus_{C \in \Sigma} \bigoplus_{a \in-\operatorname{relint}(C)} \widetilde{H}^{i-1}\left(\mathscr{C}_{\operatorname{star}_{\Sigma}(C)}\right) \otimes_{K} K(-a)
$$

as $\mathbb{Z}^{d}$-graded $K$-modules (where relint $(C)$ denotes the relative interior of $C$ with respect to the subspace topology on the vector space generated by $C$ ).

See also [4] where such a formula was given for a more general class of rings. Theorem 2.2 yields an important Cohen-Macaulay criterion for fans which generalizes the corresponding result for simplicial complexes given by Reisner in [19]:

Corollary 2.3. Let $\Sigma$ be a rational pointed fan in $\mathbb{R}^{d}$. Then $K[\Sigma]$ is Cohen-Macaulay if and only if $\widetilde{H}^{i}\left(\mathscr{C}_{\operatorname{star}_{\Sigma}(C)}\right)=0$ for all $C \in \Sigma$ and all $i<\operatorname{dim} \Sigma-1$.

Generalizing ideas of [13] and [14] we consider the complex

$$
D_{\bullet}(\Sigma): 0 \longrightarrow D_{\operatorname{dim} \Sigma} \stackrel{\partial}{\longrightarrow} D_{\operatorname{dim} \Sigma-1} \longrightarrow \cdots \longrightarrow D_{1} \stackrel{\partial}{\longrightarrow} D_{0} \longrightarrow 0,
$$

where we set

$$
D_{i}=\bigoplus_{C \in \Sigma, \operatorname{dim} C=i} K\left[C \cap \mathbb{Z}^{d}\right]
$$

for $i=0, \ldots, \operatorname{dim} \Sigma$, and the differential is the canonical projection multiplied with an incidence function $\varepsilon$ on $\Sigma$. (We refer again to [15, Section 5] for a comprehensive treatment.)

Assume that $K[\Sigma]$ is Cohen-Macaulay. Since $K[\Sigma]$ is a factor ring of a polynomial ring, the canonical module $\omega_{K[\Sigma]}$ exists. The module $\omega_{K[\Sigma]}$ is described by:

THEOREM 2.4. ([15, Corollary 5.2]) Let $\Sigma$ be a rational pointed fan in $\mathbb{R}^{d}$ with $k=\operatorname{dim} \Sigma$ and assume that $K[\Sigma]$ is Cohen-Macaulay. Then $\omega_{K[\Sigma]}$ is isomorphic to the kernel of $D_{k} \stackrel{\partial}{\rightarrow} D_{k-1}$ and the complex of $\mathbb{Z}^{d}$-graded $K[\Sigma]$-modules

$$
0 \longrightarrow \omega_{K[\Sigma]} \longrightarrow D_{k} \stackrel{\partial}{\longrightarrow} D_{k-1} \longrightarrow \cdots \longrightarrow D_{1} \stackrel{\partial}{\longrightarrow} D_{0} \longrightarrow 0
$$

is exact. 
For a cone $C \in \Sigma$ let $f_{i}\left(\operatorname{star}_{\Sigma}(C)\right)$ be the number of $i$-dimensional cones in $\operatorname{star}_{\Sigma}(C)$. We define

$$
\rho_{\Sigma}(C)=\sum_{i=0}^{\operatorname{dim} \Sigma}(-1)^{i+1} f_{i}\left(\operatorname{star}_{\Sigma}(C)\right) .
$$

Observe that the definition of $\rho$ differs from the definition of the number $\tilde{\chi}_{\Sigma}(C)$ as considered in [15] by the sign $(-1)^{\operatorname{dim} C+1}$. Hence all results cited from [15] have to take into account this factor. But we will see soon that one gets more compact formulas using $\rho_{\Sigma}(C)$ as defined above. An easy computation shows that

$$
\begin{aligned}
\rho_{\Sigma}(C) & =\sum_{i=\operatorname{dim} C-1}^{\operatorname{dim} \Sigma-1}(-1)^{i} \operatorname{dim}_{K}\left(\mathscr{C}_{\operatorname{star}_{\Sigma}(C)}^{i}\right) \\
& =\sum_{i=\operatorname{dim} C-1}^{\operatorname{dim} \Sigma-1}(-1)^{i} \operatorname{dim}_{K} \widetilde{H}^{i}\left(\mathscr{C}_{\operatorname{star}_{\Sigma}(C)}\right),
\end{aligned}
$$

so $\rho_{\Sigma}(C)$ is nothing else but the Euler characteristic of the complex $\mathscr{C}_{\text {star }_{\Sigma}(C)}$.

For a $\mathbb{Z}^{d}$-graded module $M$ we let $\operatorname{Supp}\left(M, \mathbb{Z}^{d}\right)=\left\{a \in \mathbb{Z}^{d}: M_{a} \neq 0\right\}$ be the support of $M$ in $\mathbb{Z}^{d}$.

Gorenstein toric face rings are described by the next theorem.

THEOREM 2.5. ([15, Theorem 6.2]) Let $\Sigma$ be a rational pointed fan in $\mathbb{R}^{d}$. Then $K[\Sigma]$ is Gorenstein if and only if $K[\Sigma]$ is Cohen-Macaulay and there exists $\sigma \in\left[\bigcap_{C \in \Sigma \text { maximal }} C\right] \cap \mathbb{Z}^{d}$ such that:

(i)

$$
\bigcup_{C \in \operatorname{star}_{\Sigma}(\sigma)} \operatorname{relint}(C) \cap \mathbb{Z}^{d}=\sigma+\operatorname{Supp}\left(K[\Sigma], \mathbb{Z}^{d}\right) .
$$

(ii) For all cones $C \in \Sigma$ we have

$$
\rho_{\Sigma}(C)= \begin{cases}(-1)^{\operatorname{dim} \Sigma-1} & \text { if } C \in \operatorname{star}_{\Sigma}(\sigma) \\ 0 & \text { if } C \in \Sigma(\sigma) .\end{cases}
$$

We call $\Sigma$ an Euler fan if $\Sigma$ is pure (i.e. all facets of $\Sigma$ have the same dimension), and $\rho_{\Sigma}(C)=(-1)^{\operatorname{dim} \Sigma-1}$ for all $C \in \Sigma$. We have the following characterizations of Cohen-Macaulay and Euler fans: 
Proposition 2.6. ([15, Corollary 6.5]) Let $\Sigma$ be a rational pointed fan in $\mathbb{R}^{d}$. The following statements are equivalent:

(i) $K[\Sigma]$ is Cohen-Macaulay and $\Sigma$ is an Euler fan;

(ii) $K[\Sigma]$ is Gorenstein and $\omega_{K[\Sigma]} \cong K[\Sigma]$ as $\mathbb{Z}^{d}$-graded modules;

(iii) $K[\Sigma]$ is Gorenstein and $\rho_{\Sigma}(0)=(-1)^{\operatorname{dim} \Sigma-1}$;

(iv) $K[\Sigma]$ is Cohen-Macaulay and $\widetilde{H}^{\operatorname{dim} \Sigma-1}\left(\mathscr{C}_{\operatorname{star}_{\Sigma}(C)}\right)=K$ for all $C \in \Sigma$.

\section{§3. Embedding of the canonical module as an ideal of a toric face ring}

Let $K$ be a field, and $\Sigma$ be a rational pointed fan in $\mathbb{R}^{d}$ such that $K[\Sigma]$ is a Cohen-Macaulay ring. An admissible $\mathbb{Z}$-grading on $K[\Sigma]$ is a $\mathbb{Z}$-grading such that the ring $K[\Sigma]=\bigoplus_{i \in \mathbb{N}} K[\Sigma]_{i}$ is a finitely generated positively graded $K$-algebra with $K=K[\Sigma]_{0}$ and all components $K[\Sigma]_{i}$ are direct sums of finitely many $\mathbb{Z}^{d}$-graded components. Observe that there exist fans without such gradings on $K[\Sigma]$. (See [7, Example 2.7].)

In [15, Corollary 5.3.] it was shown that in case that there exists an admissible $\mathbb{Z}$-grading on $K[\Sigma]$, then there exists a $\mathbb{Z}$-graded embedding of the canonical module $\omega_{K[\Sigma]}$ into $K[\Sigma]$. Then $\omega_{K[\Sigma]}$ can be identified with a $\mathbb{Z}$-graded ideal $I$ of $K[\Sigma]$.

In general, if $\omega_{K[\Sigma]}$ can be identified with an ideal $I$ of $K[\Sigma]$ we cannot expect that $I$ is $\mathbb{Z}^{d}$-graded, simply because it may happen that $\operatorname{dim}_{K}\left(\omega_{K[\Sigma]}\right)_{a}>1$ for some $a \in \mathbb{Z}^{d}$. See [9, Section 5.7] for such an example for a Stanley-Reisner ring. The case when $I$ is $\mathbb{Z}^{d}$-graded is however interesting and it is a natural question to characterize this situation.

THEOREM 3.1. Let $\Sigma$ be a rational pointed fan in $\mathbb{R}^{d}$ such that $K[\Sigma]$ is a Cohen-Macaulay ring. Then the following statements are equivalent:

(i) $\Sigma$ is not an Euler fan, and there exists an embedding $\omega_{K[\Sigma]} \rightarrow K[\Sigma]$ of $\mathbb{Z}^{d}$-graded $K[\Sigma]$-modules;

(ii) There exists a $(\operatorname{dim} \Sigma-1)$-dimensional subfan $\Sigma^{\prime}$ of $\Sigma$ which is Euler and Cohen-Macaulay over $K$ such that for all $C \in \Sigma$

$$
\widetilde{H}^{\operatorname{dim} \Sigma-1}\left(\mathscr{C}_{\operatorname{star}_{\Sigma}(C)}\right)= \begin{cases}0 & \text { if } C \in \Sigma^{\prime} \\ K & \text { if } C \notin \Sigma^{\prime}\end{cases}
$$


The theorem generalizes a well-known result [9, Theorem 5.7.1] for Stanley-Reisner rings. For the proof we need a special construction. We always embed $\mathbb{R}^{d}$ naturally into $\mathbb{R}^{d+1}$ onto the first $d$ coordinates and identify the basis-vectors $e_{1}, \ldots, e_{d}$ of the corresponding standard bases of $\mathbb{R}^{d}$ and $\mathbb{R}^{d+1}$ respectively. Let $C$ be a rational pointed cone in $\mathbb{R}^{d}$ and $w$ a rational point in $\mathbb{R}^{d+1} \backslash \mathbb{R}^{d}$ (e.g. $w=e_{d+1}$ ). We denote by $w * C$ the smallest (pointed) cone containing both $w$ and $C$. If $\Sigma$ is rational pointed fan in $\mathbb{R}^{d}$, we denote by $w * \Sigma$ the fan with facets $\{w * C: C$ facet of $\Sigma\}$. Clearly $w * \Sigma$ is a $(\operatorname{dim} \Sigma+1)$-dimensional fan in $\mathbb{R}^{d+1}$.

Proof. Before proving the equivalence of (i) and (ii), we observe the following. For a $\mathbb{Z}^{d}$-graded $K[\Sigma]$-module $M$ we let $M^{\vee}=\operatorname{Hom}_{K}(M, K)$ be the $\mathbb{Z}^{d}$-graded $K$-dual of $M$ which is again a naturally $\mathbb{Z}^{d}$-graded $K[\Sigma]$ module with homogeneous components $\left(M^{\vee}\right)_{a}=\operatorname{Hom}_{K}\left(M_{-a}, K\right)$ for $a \in$ $\mathbb{Z}^{d}$. It follows from the local duality theorem for $\mathbb{Z}^{d}$-graded modules (see $[9$, Theorem 3.6.19.] for the $\mathbb{Z}$-graded case) that

$$
\omega_{K[\Sigma]} \cong\left(H_{\mathfrak{m}}^{\operatorname{dim} \Sigma}(K[\Sigma])\right)^{\vee} \cong \bigoplus_{a \in \mathbb{Z}^{d}} \operatorname{Hom}_{K}\left(H_{\mathfrak{m}}^{\operatorname{dim} \Sigma}(K[\Sigma])_{-a}, K\right) .
$$

Using this fact and Theorem 2.2 we deduce that there exists an isomorphism of $\mathbb{Z}^{d}$-graded $K$-vector spaces:

$$
\omega_{K[\Sigma]} \cong \bigoplus_{C \in \Sigma} \bigoplus_{a \in \operatorname{relint}(C) \cap \mathbb{Z}^{d}} \tilde{H}^{\operatorname{dim} \Sigma-1}\left(\mathscr{C}_{\operatorname{star}_{\Sigma}(C)}\right) \otimes_{K} K(-a) .
$$

(i) $\Rightarrow$ (ii): Let $I$ be the $\mathbb{Z}^{d}$-graded ideal which is isomorphic to the image of $\omega_{K[\Sigma]} \rightarrow K[\Sigma]$. Since we assume that $\Sigma$ is not an Euler fan, it follows from Proposition 2.6 that $I \neq K[\Sigma]$. It follows from (1) that for $C \in \Sigma$ we have either

$$
\operatorname{relint}(C) \cap \mathbb{Z}^{d} \subseteq \operatorname{Supp}\left(I, \mathbb{Z}^{d}\right) \text { or } \operatorname{relint}(C) \cap \mathbb{Z}^{d} \bigcap \operatorname{Supp}\left(I, \mathbb{Z}^{d}\right)=\emptyset .
$$

We define

$$
\begin{aligned}
\Sigma^{\prime} & =\left\{C \in \Sigma: \operatorname{relint}(C) \cap \mathbb{Z}^{d} \bigcap \operatorname{Supp}\left(I, \mathbb{Z}^{d}\right)=\emptyset\right\} \\
& =\left\{C \in \Sigma: \widetilde{H}^{\operatorname{dim} \Sigma-1}\left(\mathscr{C}_{\operatorname{star}_{\Sigma}(C)}\right)=0\right\}
\end{aligned}
$$

and claim that $\Sigma^{\prime}$ is a subfan of $\Sigma$. Let $C \in \Sigma^{\prime}$ and $C^{\prime}$ be a face of $C$. Assume that $C^{\prime} \notin \Sigma^{\prime}$ and take $a, a^{\prime} \in \mathbb{Z}^{d}$ such that $a \in \operatorname{relint}(C)$ and 
$a^{\prime} \in \operatorname{relint}\left(C^{\prime}\right)$. Then $x^{a} \notin I$ and $x^{a^{\prime}} \in I$. Since $a+a^{\prime} \in \operatorname{relint}(C)$, it follows that $x^{a} x^{a^{\prime}}=x^{a+a^{\prime}} \notin I$, a contradiction since $I$ is an ideal. Thus we see that indeed $\Sigma^{\prime}$ is a subfan of $\Sigma$. Moreover, $I=\mathfrak{q}_{\Sigma^{\prime}}$ and by definition we have for all $C \in \Sigma^{\prime}$

$$
\widetilde{H}^{\operatorname{dim} \Sigma-1}\left(\mathscr{C}_{\operatorname{star}_{\Sigma}(C)}\right)= \begin{cases}0 & \text { if } C \in \Sigma^{\prime}, \\ K & \text { if } C \notin \Sigma^{\prime} .\end{cases}
$$

Since $I \neq K[\Sigma]$ we deduce $\operatorname{dim} \Sigma>0$. If $C \in \Sigma$ is a facet, then $\widetilde{H}^{\operatorname{dim} \Sigma-1}\left(\mathscr{C}_{\operatorname{star}_{\Sigma}(C)}\right) \neq 0$. For each facet $C$ of $\Sigma$ we choose $a_{C} \in \operatorname{relint}(C) \cap$ $\mathbb{Z}^{d}$. It follows from the definition of the ring structure of $K[\Sigma]$ that

$$
\sum_{C \in \Sigma \text { facet }} x^{a_{C}} \in I
$$

is a $K[\Sigma]$-regular element. $I \cong \omega_{K[\Sigma]}$ is a canonical module for $K[\Sigma]$ in the category of $K[\Sigma]$-modules. An ideal which contains a regular element has a rank, and we may use $\left[9\right.$, Proposition 3.3.18 (b)] to see that $K\left[\Sigma^{\prime}\right]=$ $K[\Sigma] / I$ is Gorenstein (in particular Cohen-Macaulay) of dimension $\operatorname{dim} \Sigma-$ 1. Applying the functor $\operatorname{Hom}_{K[\Sigma]}\left(-, \omega_{K[\Sigma]}\right)$ in the category of $\mathbb{Z}^{d}$-graded $K[\Sigma]$-modules to the exact sequence of $\mathbb{Z}^{d}$-graded $K[\Sigma]$-modules

$$
0 \longrightarrow \omega_{K[\Sigma]} \longrightarrow K[\Sigma] \longrightarrow K\left[\Sigma^{\prime}\right] \longrightarrow 0
$$

we obtain the exact sequence

$$
\begin{aligned}
0 \longrightarrow \omega_{K[\Sigma]} \longrightarrow \operatorname{Hom}_{K[\Sigma]}\left(\omega_{K[\Sigma]}, \omega_{K[\Sigma]}\right) & \longrightarrow \operatorname{Ext}_{K[\Sigma]}^{1}\left(K\left[\Sigma^{\prime}\right], \omega_{K[\Sigma]}\right) \\
& \longrightarrow \operatorname{Ext}_{K[\Sigma]}^{1}\left(K[\Sigma], \omega_{K[\Sigma]}\right) \longrightarrow \cdots
\end{aligned}
$$

where we used the facts that $\operatorname{Hom}_{K[\Sigma]}\left(K\left[\Sigma^{\prime}\right], \omega_{K[\Sigma]}\right)=0$ and $\operatorname{Hom}_{K[\Sigma]}(K[\Sigma]$, $\left.\omega_{K[\Sigma]}\right)=\omega_{K[\Sigma]}$. Furthermore we have $\operatorname{Hom}_{K[\Sigma]}\left(\omega_{K[\Sigma]}, \omega_{K[\Sigma]}\right) \cong K[\Sigma]$ and $\operatorname{Ext}_{K[\Sigma]}^{1}\left(K\left[\Sigma^{\prime}\right], \omega_{K[\Sigma]}\right) \cong \omega_{K\left[\Sigma^{\prime}\right]}$ as $\mathbb{Z}^{d}$-graded $K[\Sigma]$-modules. We also have $\operatorname{Ext}_{K[\Sigma]}^{1}\left(K[\Sigma], \omega_{K[\Sigma]}\right)=0$. (See [9] for details.)

So there exists a short exact sequence of $\mathbb{Z}^{d}$-graded $K[\Sigma]$-modules

$$
0 \longrightarrow \omega_{K[\Sigma]} \longrightarrow K[\Sigma] \longrightarrow \omega_{K\left[\Sigma^{\prime}\right]} \longrightarrow 0
$$

We conclude that $K\left[\Sigma^{\prime}\right] \cong \omega_{K\left[\Sigma^{\prime}\right]}$ as $\mathbb{Z}^{d}$-graded modules and it follows now from Proposition 2.6 that $\Sigma^{\prime}$ is an Euler fan.

(ii) $\Rightarrow($ i $): \quad \Sigma^{\prime} \neq \emptyset$ and $\widetilde{H}^{\operatorname{dim} \Sigma-1}\left(\mathscr{C}_{\operatorname{star}_{\Sigma}(C)}\right)=0$ for all $C \in \Sigma^{\prime}$. By Proposition 2.6 (iv), $\Sigma$ is not an Euler fan. 
Now we show that $\mathfrak{q}_{\Sigma^{\prime}}^{\Sigma}$ is isomorphic with the canonical module of $\Sigma$ which proves (i). Let $w$ a rational point in $\mathbb{R}^{d+1} \backslash \mathbb{R}^{d}$ and consider the rational pointed fan $\Pi=\left(w * \Sigma^{\prime}\right) \cup \Sigma$ in $\mathbb{R}^{d+1}$ whose facets are the facets of the fan $w * \Sigma^{\prime}$ and the facets of the fan $\Sigma$. (Here we consider $\Sigma$ as the fan in $\mathbb{R}^{d+1}$ induced by the embedding $\mathbb{R}^{d} \rightarrow \mathbb{R}^{d+1}$.)

We claim that $\Pi$ is Euler and $K[\Pi]$ is Cohen-Macaulay of dimension $\operatorname{dim} \Sigma$. For this we prove that for all $C \in \Pi$

$$
\widetilde{H}^{i}\left(\mathscr{C}_{\operatorname{star}_{\Pi}(C)}\right)= \begin{cases}0 & \text { if } i<\operatorname{dim} \Sigma-1, \\ K & \text { if } i=\operatorname{dim} \Sigma-1 .\end{cases}
$$

Then it follows from Theorem 2.3 that $K[\Pi]$ is Cohen-Macaulay of dimension $\operatorname{dim} \Sigma$ and using (iv) in Proposition 2.6 we can conclude that $\Pi$ is an Euler fan.

We consider the following cases:

(a) If $C \in \Sigma \backslash \Sigma^{\prime}$, then $\operatorname{star}_{\Pi}(C)=\operatorname{star}_{\Sigma}(C)$ and (2) follows from the assumption of (ii) and the Cohen-Macaulayness of $K[\Sigma]$ (using also Corollary 2.3).

(b) If $w \in C$, then $C=w * F$ where $F \in \Sigma^{\prime}$ and thus $\operatorname{star}_{\Pi}(C)$ is isomorphic as posets to $\operatorname{star}_{\Sigma^{\prime}}(F)$. Thus (2) follows since $\Sigma^{\prime}$ is Euler and Cohen-Macaulay over $K$. (One has to use again Corollary 2.3 and Proposition 2.6 applied to $K\left[\Sigma^{\prime}\right]$.)

(c) If $C \in \Sigma^{\prime}$, then $\operatorname{star}_{\Pi}(C)=\left\{w * F: F \in \operatorname{star}_{\Sigma^{\prime}}(C)\right\} \cup \operatorname{star}_{\Sigma}(C)$. Moreover, we have that $\left\{w * F: F \in \operatorname{star}_{\Sigma^{\prime}}(C)\right\} \cap \operatorname{star}_{\Sigma}(C)=\emptyset$. Set $\mathscr{C}_{\operatorname{star}_{\Sigma^{\prime}}(C)}[-1]$ to be the complex $\mathscr{C}_{\text {star }_{\Sigma^{\prime}}(C)}$ right shifted by one position (that is $\left.\mathscr{C}_{\operatorname{star}_{\Sigma^{\prime}}(C)}^{i}[-1]=\mathscr{C}_{\operatorname{star}_{\Sigma^{\prime}}(C)}^{i-1}\right)$. Then we get an exact sequence of complexes

$$
0 \longrightarrow \mathscr{C}_{\operatorname{star}_{\Sigma}(C)}^{\bullet} \longrightarrow \mathscr{C}_{\operatorname{star}_{\Pi}(C)}^{\bullet} \longrightarrow \mathscr{C}_{\operatorname{star}_{\Sigma^{\prime}}(C)}^{\bullet}[-1] \longrightarrow 0
$$

This yields the long exact cohomology sequence

$$
\cdots \longrightarrow \widetilde{H}^{i}\left(\mathscr{C}_{\operatorname{star}_{\Sigma}(C)}\right) \longrightarrow \widetilde{H}^{i}\left(\mathscr{C}_{\operatorname{star}_{\Pi}(C)}\right) \longrightarrow \widetilde{H}^{i-1}\left(\mathscr{C}_{\operatorname{star}_{\Sigma^{\prime}}(C)}\right) \longrightarrow \cdots
$$

Since $K[\Sigma]$ is Cohen-Macaulay, it follows from Corollary 2.3 that $\widetilde{H}^{i}\left(\mathscr{C}_{\operatorname{star}_{\Sigma}(C)}\right)=0$ for all $i \neq \operatorname{dim} \Sigma-1$. But $C \in \Sigma^{\prime}$ and hence also $\widetilde{H}^{\operatorname{dim} \Sigma-1}\left(\mathscr{C}_{\operatorname{star}_{\Sigma}(C)}\right)=0$. We conclude that

$$
\widetilde{H}^{i}\left(\mathscr{C}_{\operatorname{star}_{\Pi}(C)}\right) \cong \widetilde{H}^{i-1}\left(\mathscr{C}_{\operatorname{star}_{\Sigma^{\prime}}(C)}\right) \quad \text { for all } i
$$


As $\Sigma^{\prime}$ is Euler and $K\left[\Sigma^{\prime}\right]$ is Cohen-Macaulay, we immediately see that (2) holds for $\Pi$.

We have shown that $K[\Pi]$ is Cohen-Macaulay and $\Pi$ is Euler. Thus it follows from Proposition 2.6 that $K[\Pi]$ is Gorenstein and $\omega_{K[\Pi]} \cong K[\Pi]$ as $\mathbb{Z}^{d}$-graded modules. Then the $\mathbb{Z}^{d}$-graded analogue of [9, Proposition 3.6.12] implies the $\mathbb{Z}^{d}$-graded $K[\Sigma]$-module isomorphisms

$$
\omega_{K[\Sigma]} \cong \operatorname{Hom}_{K[\Pi]}(K[\Sigma], K[\Pi]) \cong \operatorname{Ann}_{K[\Pi]} \mathfrak{q}_{\Sigma}^{\Pi}
$$

The monomials in the ideal $\operatorname{Ann}_{K[\Pi]} \mathfrak{q}_{\Sigma}^{\Pi}$ of $K[\Pi]$ are precisely those $x^{a}$ with $a \in \Sigma \backslash \Sigma^{\prime}$. Thus as a $\mathbb{Z}^{d}$-graded $K[\Sigma]$-module it is isomorphic to $\mathfrak{q}_{\Sigma^{\prime}}^{\Sigma}$. Hence

$$
\omega_{K[\Sigma]} \cong \mathfrak{q}_{\Sigma^{\prime}}^{\Sigma}
$$

as desired.

Note that if the image of the embedding $\omega_{K[\Sigma]} \rightarrow K[\Sigma]$ is a principal ideal, then $K[\Sigma]$ is Gorenstein.

\section{§4. On manifolds related to rational pointed fans}

For further results on toric face rings we need some facts related to a regular cell complex induced by a rational pointed fan $\Sigma$ in $\mathbb{R}^{d}$. For this we consider the intersection of $\Sigma$ with the unit sphere $\mathbb{S}^{d-1}$ and the set $\Gamma_{\Sigma}=\left\{\operatorname{relint}(C) \cap \mathbb{S}^{d-1}: C \in \Sigma\right\}$. For a cone $C \in \Sigma$ we denote by $e_{C}=\operatorname{relint}(C) \cap \mathbb{S}^{d-1}$ the corresponding element of $\Gamma_{\Sigma}$. The elements $e_{C}$ are called open cells. Set $\Gamma_{\Sigma}^{i}=\left\{e_{C} \in \Gamma_{\Sigma}: \bar{e}_{C}\right.$ homeomorphic with $\left.\mathbb{B}^{i}\right\}$ where $\mathbb{B}^{i}$ is the $i$-dimensional ball in $\mathbb{R}^{i}$. Now define $\left|\Gamma_{\Sigma}\right|=\bigcup_{C \in \Gamma} e_{C}$. Thus $\left|\Gamma_{\Sigma}\right|$ is just the intersection of the underlying topological space $|\Sigma|=\bigcup_{C \in \Sigma} C$ of $\Sigma$ with the unit sphere $\mathbb{S}^{d-1}$. Then $\left(\left|\Gamma_{\Sigma}\right|, \Gamma_{\Sigma}\right)$ is a finite regular cell complex. The dimension of $\Gamma_{\Sigma}$ is given by $\operatorname{dim} \Gamma_{\Sigma}=\max \left\{i: \Gamma_{\Sigma}^{i} \neq \emptyset\right\}=\operatorname{dim} \Sigma-1$. An element $e_{C^{\prime}}$ is called a face of $e_{C}$ if $e_{C^{\prime}} \subset \bar{e}_{C}$, i.e. $C^{\prime}$ is a face of $C$. Note that we consider $\emptyset=e_{\{0\}}$ also as a cell in $\Gamma_{\Sigma}$.

The following result is crucial. First we need some further notations. We already considered simplicial complexes $\Delta$ on a finite vertex set $V$. The elements $F \in \Delta$ are called faces of $\Delta$. If $F \neq \emptyset$ we define the dimension of $F$ to be $\operatorname{dim} F=\# F-1$. We set $\operatorname{dim} \emptyset=-1$ and then the $\operatorname{dimension} \operatorname{dim} \Delta$ of $\Delta$ is the maximum of $\operatorname{dim} F$ for $F \in \Delta$. For $F \in \Delta$ let $\operatorname{link}_{\Delta} F$ be the set $\{G \in \Delta: F \cup G \in \Delta, F \cap G=\emptyset\}$ and call it the link of $F$. Simplicial complexes (and their faces) have geometric realizations which we usually 
denote by $|\Delta|$ and then $\Delta$ is said to be a triangulation of $|\Delta|$. We recall one important construction. Let $P$ be a finite partially ordered set (poset for short) which has a unique minimal element $\widehat{0}$ (e.g. the face poset of $\Sigma$, the face poset of $\Gamma_{\Sigma}$ or $\left.\operatorname{star}_{\Sigma} C\right)$. We denote by $\Delta(P)$ the order complex of $P \backslash\{\widehat{0}\}$ which is the simplicial complex where the faces are the chains of $P \backslash\{\widehat{0}\}$. For further definitions and results on simplicial complexes and posets see [9] and [21] respectively.

Furthermore, for a topological space $X, p \in X$ and $K$ a field we denote by $\widetilde{H}_{i}(X)$ the reduced simplicial homology with coefficients in $K$, and by $H_{i}(X, X \backslash\{p\})$ the local homology groups of $X$ at $p$ with coefficients in $K$.

LEMMA 4.1. Let $\Sigma$ be a rational pointed fan in $\mathbb{R}^{d}, 0 \neq C \in \Sigma$ and $p \in e_{C}$. Then

$$
H_{i}\left(\left|\Gamma_{\Sigma}\right|,\left|\Gamma_{\Sigma}\right| \backslash\{p\}\right) \cong \widetilde{H}^{i}\left(\mathscr{C}_{\operatorname{star}_{\Sigma} C}\right) \quad \text { for all } i
$$

Moreover,

$$
\widetilde{H}_{i}\left(\left|\Gamma_{\Sigma}\right|\right) \cong \widetilde{H}^{i}\left(\mathscr{C}_{\operatorname{star} \Sigma(0)}\right) \quad \text { for all } i
$$

Proof. Let $\mathscr{F}$ be a maximal chain $C_{1} \subset \cdots \subset C_{r}$ in the order complex $\Delta(\Sigma)$ of the face poset of $\Sigma$ such that $C_{r}=C$. Observe that then $\operatorname{dim} \mathscr{F}=\operatorname{dim} C-1$ and $\operatorname{link}_{\Delta(\Sigma)} \mathscr{F}=\Delta(\operatorname{star} C)$. Note further that $\Delta(\Sigma)$ is a triangulation of $\Gamma_{\Sigma}$ (see [3, Proposition 4.7.8]) and therefore has the geometric realization $|\Delta(\Sigma)|=\left|\Gamma_{\Sigma}\right|$. In particular, we may assume $p \in|\Delta(\Sigma)|$. We compute

$$
\begin{aligned}
\widetilde{H}^{i}\left(\mathscr{C}_{\operatorname{star}_{\Sigma} C}\right) & \cong \widetilde{H}^{i-\operatorname{dim} C}\left(\Delta\left(\operatorname{star}_{\Sigma} C\right)\right) \\
& \cong \widetilde{H}_{i-\operatorname{dim} C}\left(\Delta\left(\operatorname{star}_{\Sigma} C\right)\right) \\
& =\widetilde{H}_{i-\operatorname{dim} C}\left(\operatorname{link}_{\Delta(\Sigma)} \mathscr{F}\right) \\
& \cong H_{i}(|\Delta(\Sigma)|,|\Delta(\Sigma)| \backslash\{p\}) \\
& \cong H_{i}\left(\left|\Gamma_{\Sigma}\right|,\left|\Gamma_{\Sigma}\right| \backslash\{p\}\right) .
\end{aligned}
$$

Here the first isomorphism was shown in [15, Lemma 4.6]. The second isomorphism follows since we take (co-)homology with coefficients in $K$. The third equality follows from the observations above. For the fourth isomorphism see $\left[9\right.$, Lemma 5.4.5]. Here note that if $\operatorname{star}_{\Sigma} C=\{C\}$ (that is $\left.\operatorname{link}_{\Delta(\Sigma)} \mathscr{F}=\emptyset\right)$, then

$$
\widetilde{H}^{i}\left(\mathscr{C}_{\operatorname{star}_{\Sigma} C}\right) \cong \widetilde{H}_{i-\operatorname{dim} C}\left(\operatorname{link}_{\Delta(\Sigma)} \mathscr{F}\right) \cong \begin{cases}K & \text { if } i=\operatorname{dim} C-1 \\ 0 & \text { otherwise }\end{cases}
$$


The last isomorphism follows from [3, Proposition.4.7.8].

The supplement concerning the reduced simplicial homology is obvious since we have that $\Delta\left(\operatorname{star}_{\Sigma}(0)\right)=\Delta(\Sigma)$.

We immediately get that the Cohen-Macaulay property of $K[\Sigma]$ only depends on the topology of $\left|\Gamma_{\Sigma}\right|$. This generalizes the corresponding result for simplicial complexes given by Munkres in [18].

Corollary 4.2. Let $\Sigma$ be a $k$-dimensional rational pointed fan in $\mathbb{R}^{d}$. The following conditions are equivalent:

(a) $K[\Sigma]$ is Cohen-Macaulay;

(b) For all $p \in\left|\Gamma_{\Sigma}\right|$ and all $i<k-1$ one has

$$
\widetilde{H}_{i}\left(\left|\Gamma_{\Sigma}\right|\right)=H_{i}\left(\left|\Gamma_{\Sigma}\right|,\left|\Gamma_{\Sigma}\right| \backslash\{p\}\right)=0 .
$$

Moreover, if the equivalent conditions are satisfied, then $\Sigma$ is Euler (and $K[\Sigma]$ is Gorenstein) if and only if for all $p \in\left|\Gamma_{\Sigma}\right|$

$$
\widetilde{H}_{k-1}\left(\left|\Gamma_{\Sigma}\right|\right)=H_{k-1}\left(\left|\Gamma_{\Sigma}\right|,\left|\Gamma_{\Sigma}\right| \backslash\{p\}\right)=K .
$$

In particular:

(i) If $\left|\Gamma_{\Sigma}\right|$ is homeomorphic to a $(k-1)$-dimensional sphere, then $\Sigma$ is Euler and $K[\Sigma]$ is Gorenstein.

(ii) If $\left|\Gamma_{\Sigma}\right|$ is homeomorphic to a $(k-1)$-dimensional ball, then $K[\Sigma]$ is Cohen-Macaulay.

Proof. $\quad(\mathrm{a}) \Leftrightarrow(\mathrm{b})$ : This is Corollary 2.3 where one replaces $\widetilde{H}^{i}\left(\mathscr{C}_{\operatorname{star}_{\Sigma} C}\right)$ according to Lemma 4.1. Using Proposition 2.6, (i) $\Leftrightarrow($ iv), the remark concerning the Euler property follows.

(i): If $\left|\Gamma_{\Sigma}\right|$ is homeomorphic to a $(k-1)$-dimensional sphere then

$$
\widetilde{H}_{i}\left(\left|\Gamma_{\Sigma}\right| ; K\right)=H_{i}\left(\left|\Gamma_{\Sigma}\right|,\left|\Gamma_{\Sigma}\right| \backslash\{p\}\right)= \begin{cases}K & \text { if } i=k-1 \\ 0 & \text { otherwise. }\end{cases}
$$

for all $p \in\left|\Gamma_{\Sigma}\right|$ (independent of the field $K$ ).

(ii): The second statement is shown analogously by observing that the local homology $H_{i}\left(\left|\Gamma_{\Sigma}\right|,\left|\Gamma_{\Sigma}\right| \backslash\{p\}\right)=0$ for $i \neq k-1$ if $\left|\Gamma_{\Sigma}\right|$ is homeomorphic to a ball. (We have that $H_{k-1}\left(\left|\Gamma_{\Sigma}\right|,\left|\Gamma_{\Sigma}\right| \backslash\{p\}\right)=0$ if $p$ is a boundary point. Thus we only get the Cohen-Macaulay property.) 
Next we give an application of Theorem 3.1. For simplicial complexes the next theorem is due to Hochster (see [9, Theorem 5.7.2] or [22, Theorem $7.3])$.

THEOREM 4.3. Let $\Sigma$ be a rational pointed fan in $\mathbb{R}^{d}$ such that $K[\Sigma]$ is a Cohen-Macaulay ring. Assume that $X=\left|\Gamma_{\Sigma}\right|$ is a manifold with a nonempty boundary $\partial X$. Further let $\Sigma^{\prime}$ be the subfan of $\Sigma$ such that $\partial X=\left|\Gamma_{\Sigma^{\prime}}\right|$. Then the following conditions are equivalent:

(i) $\omega_{K[\Sigma]} \cong \mathfrak{q}_{\Sigma^{\prime}}^{\Sigma}$ as $\mathbb{Z}^{d}$-graded $K[\Sigma]$-modules;

(ii) $\Sigma^{\prime}$ is Euler and $K\left[\Sigma^{\prime}\right]$ is Cohen-Macaulay.

Proof. (i) $\Rightarrow$ (ii): Assume that $\Sigma$ is Euler. Then $K[\Sigma]$ is Gorenstein and $K[\Sigma]=\omega_{K[\Sigma]}=\mathfrak{q}_{\Sigma^{\prime}}^{\Sigma}$ would be a contradiction to the fact that $\Sigma^{\prime}$ is non-trivial. Now (ii) was shown in (the proof of) Theorem 3.1.

(ii) $\Rightarrow$ (i): In order to use Theorem 3.1 we have to check that for all $C \in \Sigma$

$$
\widetilde{H}^{\operatorname{dim} \Sigma-1}\left(\mathscr{C}_{\operatorname{star}_{\Sigma}(C)}\right)= \begin{cases}0 & \text { if } C \in \Sigma^{\prime} \\ K & \text { if } C \notin \Sigma^{\prime}\end{cases}
$$

We have to distinguish two cases:

(a) Let $\{0\} \neq C \in \Sigma$ and choose $p \in e_{C}$. We deduce that

$$
\widetilde{H}^{\operatorname{dim} \Sigma-1}\left(\mathscr{C}_{\operatorname{star}_{\Sigma}(C)}\right) \cong H_{\operatorname{dim} \Sigma-1}\left(\left|\Gamma_{\Sigma}\right|,\left|\Gamma_{\Sigma}\right| \backslash\{p\}\right)= \begin{cases}0 & \text { if } p \in \partial X \\ K & \text { if } p \notin \partial X\end{cases}
$$

The first isomorphism follows by Lemma 4.1, while the last equality is implied by the definition of a manifold with boundary. Now $p \in \partial X$ if and only if $C \in \Sigma^{\prime}$ and thus we are done in this case.

(b) Let $C=\{0\} \in \Sigma^{\prime}$. Then we have by Lemma 4.1 that

$$
\widetilde{H}^{\operatorname{dim} \Sigma-1}\left(\mathscr{C}_{\operatorname{star}_{\Sigma}(C)}\right) \cong H_{\operatorname{dim} \Sigma-1}\left(\left|\Gamma_{\Sigma}\right|\right)
$$

That $H_{\operatorname{dim} \Sigma-1}\left(\left|\Gamma_{\Sigma}\right|\right)=0$ was shown in the proof of [9, Theorem 5.7.2] applied to the simplicial complex $\Delta(\Sigma)$ with geometric realization $X=\left|\Gamma_{\Sigma}\right|$. 


\section{$\S 5$. Shellability conditions and their algebraic properties}

In [15] the authors studied the notion of a pure shellable fan and nonpure shellable fan respectively, which imply that the corresponding toric face ring is Cohen-Macaulay and sequentially Cohen-Macaulay respectively. If the fan is the fan associated to a simplicial complex, then these notions coincide with the well-known definitions of pure shellable simplicial complexes and non-pure shellable simplicial complexes respectively. For simplicial complexes there exist various equivalent definitions of shellability.

The goal of this section is to present several shellability related notions for a rational pointed fan in $\mathbb{R}^{d}$. Some of them coincide in the "simplicial case".

Let $\Sigma$ be a rational pointed fan in $\mathbb{R}^{d}$. In the following $\partial C$ denotes the boundary of a cone $C \in \Sigma$. Recall that the fans $\operatorname{Fan}(C)$ and $\operatorname{Fan}(\partial C)$ are the set of faces of $C$ and $\partial C$ respectively. Observe that they are subfans of $\Sigma$. At first we give the definition of shellability as considered in [15] (which is motivated by the results of [1] and [2]).

Definition 5.1. A linear ordering $C_{1}, \ldots, C_{s}$ of the facets of $\Sigma$ is called a shelling of $\Sigma$ if either $\operatorname{dim} \Sigma=0$ (and thus there is just one facet and that is a point), or $\operatorname{dim} \Sigma \geq 1$ and the following two conditions are satisfied:

(i) $\operatorname{Fan}\left(\partial C_{1}\right)$ has a shelling.

(ii) For $1<j \leq s$ there exists a shelling $D_{1}^{j}, \ldots, D_{t_{j}}^{j}$ of the (pure) fan $\operatorname{Fan}\left(\partial C_{j}\right)$ such that $\bigcup_{i=1}^{j-1} \operatorname{Fan}\left(C_{i}\right) \cap \operatorname{Fan}\left(C_{j}\right)$ is not empty and equals $\bigcup_{l=1}^{r_{j}} \operatorname{Fan}\left(D_{l}^{j}\right)$ for some integer $r_{j}$ with $1 \leq r_{j} \leq t_{j}$.

$\Sigma$ is called shellable if it has a shelling.

Remark 5.2.

(i) Usually one considers only the case that $\Sigma$ is pure, i.e. all facets of $\Sigma$ have the same dimension. But in this section we allow the fans also to be non-pure and all our shellings are non-pure shellings in the terminology of [15] if not otherwise stated.

(ii) By Bruggesser-Mani condition (i) of the above definition is in fact redundant.

(iii) One can weaken this definition in an obvious way by not asking that the shelling of the fan in (ii) is the beginning of a shelling of $\operatorname{Fan}\left(\partial\left(C_{j}\right)\right)$ and gets a weaker notion of shellability, but we do not stress this point in this paper. Instead we consider semishellings defined as below. 
The next definition was inspired by [10]. (See also [16].)

Definition 5.3. A semishelling of $\Sigma$ is a linear ordering $C_{1}, \ldots, C_{s}$ of the facets of $\Sigma$ such that either $\operatorname{dim} \Sigma=0$, or $\left|\Gamma_{\bigcup_{i=1}^{j-1} \operatorname{Fan}\left(C_{i}\right) \cap \operatorname{Fan}\left(C_{j}\right)}\right|$ is homeomorphic either to a $\left(\operatorname{dim} C_{j}-2\right)$-dimensional ball or sphere for all $1<j \leq s$. The fan $\Sigma$ is called semishellable if it has a semishelling.

Of course we have:

COROLlary 5.4. Let $\Sigma$ be a rational pointed fan in $\mathbb{R}^{d}$. If $\Sigma$ is shellable, then $\Sigma$ is semishellable.

Proof. Assume that $\operatorname{dim} \Sigma>0$ and there is a shelling $C_{1}, \ldots, C_{s}$ of the facets of $\Sigma$. Now observe that the shellability of $\Sigma$ is equivalent to the fact that the regular cell complex $\Gamma_{\Sigma}$ is shellable in the sense of [3, Section 4.7] with shelling order $e_{C_{1}}, \ldots, e_{C_{s}}$. Note that $\left|\Gamma_{\mathrm{Fan}\left(\partial C_{j}\right)}\right|$ is a shellable $\left(\operatorname{dim} C_{j}-\right.$ 2 )-sphere for every $1 \leq j \leq s$. It follows now from [3, Proposition 4.7.26 (i), (ii)] that $\left|\Gamma_{\bigcup_{i=1}^{j-1} \operatorname{Fan}\left(C_{i}\right) \cap \operatorname{Fan}\left(C_{j}\right)}\right|$ is either homeomorphic to a $\left(\operatorname{dim} C_{j}-2\right)$ dimensional ball or sphere.

Semishellability has the following nice algebraic consequence.

THEOREM 5.5. Let $\Sigma$ be a rational pointed fan in $\mathbb{R}^{d}$. If $\Sigma$ is pure semishellable, then $K[\Sigma]$ is Cohen-Macaulay (independent of char $K$ ).

Proof. Using Corollary 4.2 the proof of [15, Theorem 3.2] can be easily modified to show that $K[\Sigma]$ is Cohen-Macaulay.

Semishellability has also some consequences related to canonical modules.

Remark 5.6. Let $\Sigma$ be a rational pointed semishellable fan in $\mathbb{R}^{d}$. Then one can show that the toric face ring $K\left[\bigcup_{i=1}^{j-1} \operatorname{Fan}\left(C_{i}\right) \cap \operatorname{Fan}\left(C_{j}\right)\right]$ is CohenMacaulay and $\omega_{\bigcup_{i=1}^{j-1} \operatorname{Fan}\left(C_{i}\right) \cap \operatorname{Fan}\left(C_{j}\right)}$ is isomorphic to a $\mathbb{Z}^{d}$-graded ideal of $K\left[\bigcup_{i=1}^{j-1} \operatorname{Fan}\left(C_{i}\right) \cap \operatorname{Fan}\left(C_{j}\right)\right]$ for all $1<j \leq s$. Moreover, consider the fan $\Omega_{j}=\bigcup_{l=r_{j}+1}^{t_{j}} \operatorname{Fan}\left(D_{l}^{j}\right)$. Then $\Omega_{j}=\emptyset$ or $\omega_{K\left[\Omega_{j}\right]}$ is isomorphic to a $\mathbb{Z}^{d}$-graded ideal of $K\left[\Omega_{j}\right]$ for all $1<j \leq s$.

Note that even weaker assumptions lead to the same results. 
Remark 5.7. One can weaken the definition of a semishellable fan by asking only that

$$
\widetilde{H}_{k}\left(\left|\Gamma_{\bigcup_{i=1}^{j-1} \operatorname{Fan}\left(C_{i}\right) \cap \operatorname{Fan}\left(C_{j}\right)}\right| ; K\right)= \begin{cases}0 \text { or } K & \text { if } 0 \leq k=\operatorname{dim} C_{j}-2, \\ 0 & \text { otherwise. }\end{cases}
$$

Theorem 5.5 and Remark 5.6 hold also in this context, and one may also give a converse for Remark 5.6.

Next we consider a stronger property than shellability. For this we recall the following definition which is due to Dress [11].

Definition 5.8. Let $R$ be a Noetherian ring and $M$ be a finitely generated $R$-module. A finite filtration

$$
0=M_{0} \subset M_{1} \subset \cdots \subset M_{r}=M
$$

of submodules of $M$ is called a prime filtration, if for every $1 \leq i \leq r$ there is an isomorphism $M_{i} / M_{i-1} \cong R / P_{i}$ for some prime ideal $P_{i}$ of $R$. A prime filtration is called clean if its set of corresponding prime ideals is equal to $\operatorname{Min}(\operatorname{Supp} M)$. The module $M$ is called clean, if $M$ admits a clean filtration.

Observe that there always exists a prime filtration, but clean filtrations may not exist. For simplicial complexes, cleanness is the algebraic counterpart of shellability as defined above. Next we want to study when $K[\Sigma]$ is clean for some rational pointed fan $\Sigma$. It is easy to see that if $K[\Sigma]$ is clean, then $\Sigma$ is shellable. However, cleanness is a stronger property.

THEOREM 5.9. Let $\Sigma$ be a rational pointed fan in $\mathbb{R}^{d}$. Then $K[\Sigma]$ is clean if and only if the following conditions are satisfied:

(i) $\Sigma$ is (non-pure) shellable with shelling $C_{1}, \ldots, C_{s}$.

(ii) For $1<j \leq s$ there exists $\gamma_{j} \in C_{j} \cap \mathbb{Z}^{d}$ such that

$$
\operatorname{star}_{\operatorname{Fan}\left(C_{j}\right)}\left(\gamma_{j}\right)=\operatorname{Fan}\left(C_{j}\right) \backslash\left(\bigcup_{i=1}^{j-1} \operatorname{Fan}\left(C_{i}\right)\right)
$$

$$
\bigcup_{D \in \operatorname{star}_{\operatorname{Fan}\left(C_{j}\right)}\left(\gamma_{j}\right)} \operatorname{relint}(D) \cap \mathbb{Z}^{d}=\gamma_{j}+C_{j} \cap \mathbb{Z}^{d} .
$$


In particular, the cleanness property does not depend on char $K$.

Proof. First assume that $K[\Sigma]$ is clean. The minimal prime ideals of $K[\Sigma]$ are exactly the $\mathbb{Z}^{d}$-graded prime ideals $\mathfrak{p}_{C}$ for the maximal cones $C$ of $\Sigma$. Since $K[\Sigma]$ is reduced, it follows from the last remarks in [11, Section 3] that a clean prime filtration of $K[\Sigma]$ is necessarily of the form

$$
0=\bigcap_{i=1}^{s} \mathfrak{p}_{C_{i}} \subset \bigcap_{i=1}^{s-1} \mathfrak{p}_{C_{i}} \subset \cdots \subset \mathfrak{p}_{C_{1}} \subset K[\Sigma]
$$

where $C_{1}, \ldots, C_{s}$ are the maximal cones of $\Sigma$, and there exist $\gamma_{j} \in C_{j} \cap \mathbb{Z}^{d}$ such that

$$
\bigcap_{i=1}^{j-1} \mathfrak{p}_{C_{i}} / \bigcap_{i=1}^{j} \mathfrak{p}_{C_{i}} \cong K\left[\operatorname{Fan}\left(C_{j}\right)\right]\left(-\gamma_{j}\right) \quad \text { for } \quad 1<j \leq s .
$$

Consider the fans $\Pi_{j}=\bigcup_{i=1}^{j-1}\left[\operatorname{Fan}\left(C_{i}\right) \cap \operatorname{Fan}\left(C_{j}\right)\right]$ for $j=2, \ldots, s$. Then

$$
\bigcap_{i=1}^{j-1} \mathfrak{p}_{C_{i}} / \bigcap_{i=1}^{j} \mathfrak{p}_{C_{i}}=\mathfrak{q}_{\Pi_{j}}^{K\left[\operatorname{Fan}\left(C_{j}\right)\right]} \subseteq K\left[\operatorname{Fan}\left(C_{j}\right)\right] \quad \text { for } \quad 1<j \leq s,
$$

so $\mathfrak{q}_{\Pi_{j}}^{K\left[\operatorname{Fan}\left(C_{j}\right)\right]} \cong K\left[\operatorname{Fan}\left(C_{j}\right)\right]\left(-\gamma_{j}\right)=x^{\gamma_{j}} K\left[\operatorname{Fan}\left(C_{j}\right)\right]$. Let $D \in \operatorname{Fan}\left(C_{j}\right)$. Then

$$
D \notin \Pi_{j} \Longleftrightarrow \operatorname{relint}(D) \cap \mathbb{Z}^{d} \subseteq \operatorname{Supp}\left(\mathfrak{q}_{\Pi_{j}}^{K\left[\operatorname{Fan}\left(C_{j}\right)\right]}, \mathbb{Z}^{d}\right) .
$$

Assume $\gamma_{j} \in D$. Then $a+\gamma_{j} \in \operatorname{relint}(D)$ for all $a \in \operatorname{relint}(D)$. It follows that $\left(\operatorname{relint}(D) \cap \mathbb{Z}^{d}\right) \subseteq \operatorname{Supp}\left(K\left[\operatorname{Fan}\left(C_{j}\right)\right]\left(-\gamma_{j}\right), \mathbb{Z}^{d}\right)$. Clearly $\gamma_{j} \notin D$ implies $K\left[\operatorname{Fan}\left(C_{j}\right)\right]\left(-\gamma_{j}\right) \subset \mathfrak{p}_{D}^{K\left[\operatorname{Fan}\left(C_{j}\right)\right]}$, so we have $\left(D \cap \mathbb{Z}^{d}\right) \cap \operatorname{Supp}\left(K\left[\operatorname{Fan}\left(C_{j}\right)\right]\right.$ $\left.\left(-\gamma_{j}\right), \mathbb{Z}^{d}\right)=\emptyset$. We conclude that

$$
D \in \operatorname{star}_{\operatorname{Fan}\left(C_{j}\right)}\left(\gamma_{j}\right) \Longleftrightarrow \operatorname{relint}(D) \cap \mathbb{Z}^{d} \subseteq \operatorname{Supp}\left(\mathfrak{q}_{\Pi_{j}}^{K\left[\operatorname{Fan}\left(C_{j}\right)\right]}, \mathbb{Z}^{d}\right)
$$

which implies (ii) (a), and (ii) (b)

$$
\bigcup_{D \in \operatorname{star}_{\operatorname{Fan}_{\left(C_{j}\right)}\left(\gamma_{j}\right)}} \operatorname{relint}(D) \cap \mathbb{Z}^{d}=\operatorname{Supp}\left(\mathfrak{q}_{\Pi_{j}}^{K\left[\operatorname{Fan}\left(C_{j}\right)\right]}, \mathbb{Z}^{d}\right)=\gamma_{j}+C_{j} \cap \mathbb{Z}^{d}
$$

It remains to show (i). At first observe that $\operatorname{Fan}\left(\partial C_{j}\right)$ has a shelling because this is equivalent to the fact the boundary of a cross-section polytope of 
$C_{j}$ has a shelling and this is well-known by Bruggesser-Mani. Using the notation introduced so far we have to prove for $1<j \leq s$ that $\Pi_{j}=$ $\bigcup_{l=1}^{r_{j}} \operatorname{Fan}\left(D_{l}^{j}\right)$ where $D_{1}^{j}, \ldots, D_{t_{j}}^{j}$ is a shelling of the fan $\operatorname{Fan}\left(\partial C_{j}\right)$ and $1 \leq$ $r_{j} \leq t_{j}$.

If $\gamma_{j} \in \operatorname{relint}\left(C_{j}\right)$, then $\Pi_{j}=\operatorname{Fan}\left(\partial C_{j}\right)$ and the assertion follows again directly from Bruggesser-Mani. Assume that $\gamma_{j} \notin \operatorname{relint}\left(C_{j}\right)$. Let $P_{j}$ be a cross-section polytope of $C_{j}$ such that $\gamma_{j} \in P_{j}$. It is possible to choose a point $x$ outside $P_{j}$ near $\gamma_{j}$ in general position with respect to $P_{j}$ (i.e. $x$ does not belong to an irredundant hyperplanes defining $P_{j}$ ) such that the facets of $P_{j}$ which correspond to the maximal cones in $\operatorname{star}_{\operatorname{Fan}\left(C_{j}\right)}\left(\gamma_{j}\right) \backslash\left\{C_{j}\right\}$ are exactly the visible facets from $x$. (A facet is visible if for all points $y$ on that facet the line segment between $y$ and $x$ does intersect $P_{j}$ only in $y$.) Now it follows from [24, Theorem 8.12], that there exists a (line) shelling of $P_{j}$ such that the facets induced by the maximal cones in $\operatorname{star}_{\operatorname{Fan}\left(C_{j}\right)}\left(\gamma_{j}\right) \backslash\left\{C_{j}\right\}$ are the last ones. This induces a shelling of $\partial C_{j}$ where the maximal cones in $\operatorname{star}_{\operatorname{Fan}\left(C_{j}\right)}\left(\gamma_{j}\right) \backslash\left\{C_{j}\right\}$ are the last ones. Hence the other ones which correspond to those cones in $\Pi_{j}$ are the first ones. Thus we have proved (i).

Now assume that (i) and (ii) holds. Consider the filtration of $K[\Sigma]$

$$
0=\bigcap_{i=1}^{s} \mathfrak{p}_{C_{i}} \subset \bigcap_{i=1}^{s-1} \mathfrak{p}_{C_{i}} \subset \cdots \subset \mathfrak{p}_{C_{1}} \subset K[\Sigma]
$$

induced by the shelling of (i) and the fans $\Pi_{j}$ as above. Then

$$
\bigcap_{i=1}^{j-1} \mathfrak{p}_{C_{i}} / \bigcap_{i=1}^{j} \mathfrak{p}_{C_{i}}=\mathfrak{q}_{\Pi_{j}}^{K\left[\operatorname{Fan}\left(C_{j}\right)\right]} \subseteq K\left[\operatorname{Fan}\left(C_{j}\right)\right] \quad \text { for } \quad 1<j \leq s .
$$

It follows from (ii) that $\operatorname{Supp}\left(\mathfrak{q}_{\Pi_{j}}^{K\left[\operatorname{Fan}\left(C_{j}\right)\right]}, \mathbb{Z}^{d}\right)=\operatorname{Supp}\left(x^{\gamma_{j}} K\left[\operatorname{Fan}\left(C_{j}\right)\right], \mathbb{Z}^{d}\right)$, so the two ideals coincide. But $x^{\gamma_{j}} K\left[\operatorname{Fan}\left(C_{j}\right)\right] \cong K\left[\operatorname{Fan}\left(C_{j}\right)\right]\left(-\gamma_{j}\right) \cong$ $\left(K[\Sigma] / \mathfrak{p}_{C_{j}}\right)\left(-\gamma_{j}\right)$, hence $K[\Sigma]$ is clean.

EXAMPLE 5.10. It follows from Theorem 5.9 that if $K[\Sigma]$ is clean, then $\Sigma$ is shellable. As noted above, it is known that for a simplicial complex $\Delta$ the converse is also true: If $\Delta$ is shellable, then the Stanley-Reisner ring $K[\Delta]$ is clean. (See Dress [11].) In fact, for simplicial complexes condition (ii) is always satisfied (therefore the result of Dress is contained in Theorem 5.9). For toric face rings in general the converse does not hold. Indeed, e.g. consider the fan $\Sigma$ with facets $C_{1}, C_{2} \subset \mathbb{R}^{2}$ where

$$
C_{1}=\operatorname{cn}((0,1),(2,1)) \quad \text { and } \quad C_{2}=\operatorname{cn}((0,1),(-2,1)) .
$$


Then it is easy to see that $\Sigma$ is shellable. The ring $K[\Sigma]$ is not clean because it is not possible to find $\gamma_{j}$ as given in Theorem 5.9.

Finally, one more remark on canonical modules.

Remark 5.11. Let $\Sigma$ be a rational pointed fan in $\mathbb{R}^{d}$ and let $K[\Sigma]$ be a clean ring. Let $C_{1}, \ldots, C_{s}$ be the shelling of $\Sigma$ induced by a clean prime filtration of $K[\Sigma]$. By definition, for $1<j \leq s$ there exists a shelling $D_{1}^{j}, \ldots, D_{t_{j}}^{j}$ of the (pure) fan $\operatorname{Fan}\left(\partial C_{j}\right)$ such that $\bigcup_{i=1}^{j-1}\left[\operatorname{Fan}\left(C_{i}\right) \cap\right.$ $\left.\operatorname{Fan}\left(C_{j}\right)\right]=\bigcup_{l=1}^{r_{j}} \operatorname{Fan}\left(D_{l}^{j}\right)$ for some $r_{j}$ with $1 \leq r_{j} \leq t_{j}$. Consider the fan $\Omega_{j}=\bigcup_{l=r_{j}+1}^{t_{j}} \operatorname{Fan}\left(D_{l}^{j}\right)$. Then $\Omega_{j}=\emptyset$ or $K\left[\Omega_{j}\right]$ is Gorenstein for all $1<j \leq s$ (independent of char $K$ ). That is, $\Omega_{j}=\emptyset$ or $\omega_{K\left[\Omega_{j}\right]}$ is a principal ideal of $K\left[\Omega_{j}\right]$ for all $1<j \leq s$.

Compare the Remarks 5.6 and 5.11. They provide a way to distinguish between shellable and clean fans.

\section{REFERENCES}

[1] A. Björner and M. L. Wachs, Shellable nonpure complexes and posets. I, Trans. Am. Math. Soc., 348 (1996), no. 4, 1299-1327.

[2] A. Björner and M. L. Wachs, Shellable nonpure complexes and posets. II, Trans. Am. Math. Soc., 349 (1997), no. 10, 3945-3975.

[3] A. Björner, M. Las Vergnas, B. Sturmfels, N. White and G. Ziegler, Oriented matroids. 2nd ed., Encyclopedia of Mathematics and Its Applications 46, Cambridge University Press, 1999.

[4] M. Brun, W. Bruns and T. Römer, Cohomology of partially ordered sets and local cohomology of section rings, Adv. Math., 208 (2007), no. 1, 210-235.

[5] M. Brun and T. Römer, Subdivisions of toric complexes, J. Algebr. Comb., 21 (2005), no. $4,423-448$.

[6] M. Brun and T. Römer, On algebras associated to partially ordered sets, to appear in Math. Scand.

[7] W. Bruns and J. Gubeladze, Polyhedral algebras, arrangements of toric varieties, and their groups, Adv. Stud. Pure Math., 33 (2001), 1-51.

[8] W. Bruns and J. Gubeladze, Polytopes, rings, and K-theory, preprint (2008).

[9] W. Bruns and J. Herzog, Cohen-Macaulay rings, Rev. ed., Cambridge Studies in Advanced Mathematics 39, Cambridge University Press, 1998.

[10] G. Danaraj and V. Klee, Shellings of spheres and polytopes, Duke Math. J., 41 (1974), 443-451.

[11] A. Dress, A new algebraic criterion for shellability, Beitr. Alg. Geo., 34 (1993), 4555 . 
[12] G. Fløystad, Cohen-Macaulay cell complexes, Algebraic and geometric combinatorics (C. A. Athanasiadis et al., eds.), Contemporary Mathematics 423, American Mathematical Society, 2006, pp. 205-220.

[13] H. G. Gräbe, The canonical module of a Stanley-Reisner ring, J. Algebra, 86 (1984), 272-281.

[14] H. G. Gräbe, A dualizing complex for Stanley-Reisner rings, Math. Proc. Camb. Philos. Soc., 96 (1984), 203-212.

[15] B. Ichim and T. Römer, On toric face rings, J. Pure Appl. Algebra, 210 (2007), no. $1,249-266$.

[16] P. McMullen and G. C. Shephard, Convex polytopes and the upper bound conjecture, London Mathematical Society Lecture Note Series 3, Cambridge University Press, 1971.

[17] E. Miller, Cohen-Macaulay quotients of normal semigroup rings via irreducible resolutions, Math. Research Letters, 9 (2002), 117-128.

[18] J. Munkres, Topological results in combinatorics, Michigan Math. J., 31 (1984), 113 128.

[19] G. A. Reisner, Cohen-Macaulay quotients of polynomial rings, Adv. Math., 21 (1976), $30-49$.

[20] R. P. Stanley, Generalized h-vectors, intersection cohomology of toric varieties, and related results, Commutative algebra and combinatorics, Adv. Stud. Pure Math., 11 (1987), 187-213.

[21] R. P. Stanley, Enumerative combinatorics. Vol. 1, Paperback ed., Cambridge Studies in Advanced Mathematics 49, Cambridge, Cambridge University Press, 1999.

[22] R. P. Stanley, Combinatorics and commutative algebra, 2nd ed., Progress in Mathematics 41, Birkhäuser, 2005.

[23] K. Yanagawa, Notes on C-graded modules over an affine semigroup ring $K[C]$, preprint math.AC/0506457.

[24] G. M. Ziegler, Lectures on polytopes, Springer, 1994.

Bogdan Ichim

Universität Osnabrück

FB Mathematik/Informatik

49069 Osnabrück

Germany

Institute of Mathematics

C.P. 1-764, 70700 Bucharest

Romania

bogdan.ichim@math.uos.de, bogdan.ichim@imar.ro 
Tim Römer

Universität Osnabrück

FB Mathematik/Informatik

49069 Osnabrück

Germany

troemer@uos.de 